\title{
Delay-Guaranteed Admission Control for LAA Coexisting with $\mathrm{WiFi}$
}

\author{
Weiwei Zhou, Gordon J. Sutton, J. Andrew Zhang, Ren Ping Liu, and Su Pan
}

\begin{abstract}
Licensed-assisted-access (LAA) is used to extend the LTE link into the unlicensed band. How to guarantee the quality-of-service $(\mathrm{QoS})$ for LTE devices in the unlicensed band is a challenging problem due to the listen-before-talk contention access in $5 \mathrm{GHz}$ unlicensed bands. In this paper, we quantitatively analyze the MAC delay for tagged LAA eNBs and propose a delay-guaranteed admission control scheme. We consider the freezing time of busy slots caused by collision or successful transmission, and introduce the exponential backoff mechanism for delay analysis. Validated by simulation results, our method provides important insights into the system admission performance and fairness of access.
\end{abstract}

Index Terms-Admission Control, Licensed-assisted Access, Listen Before Talk.

\section{INTRODUCTION}

Licensed-Assisted Access (LAA), which adopts the ListenBefore-Talk (LBT) mechanism as the medium access control (MAC) protocol, is supported by 3GPP and many network operators [1] to meet the exponentially growing demand for data traffic. Coexistence between LAA eNBs and WiFi stations in the unlicensed spectrum brings great challenges to both LAA and WiFi. The LAA transmission is dependent on the channel state. The busier the channel is, the longer MAC delay the LAA will suffer, as the LAA counter freeze and suspend transmitting when the channel is busy. Without proper admission control (AC) and resource optimization, the access of excessive LAA eNBs can lead to high collision probabilities, thus degrading the performance of WiFi stations (devices or users) severely, as well as that of LAA eNBs themselves.

Among the limited work on AC for LAA, none has considered a comprehensive WiFi and LAA access model including both exponential backoff and delay, which can have significant impact on the efficiency of AC. In [2], the delay is analyzed for LAA systems with an adjustable LAA contention window, for a single backoff stage. In [3], an adaptive backoff window, again with a single backoff stage, is considered for AC, but it aims for fair coexistence, rather than for guaranteeing delay. In [4], the backoff process is studied for both random contention window size and binary exponential access scheme with different sizes, but the delay is not considered.

In this paper, we propose an AC scheme for coexisting LAA and $\mathrm{WiFi}$ systems that considers a comprehensive backoff model, particularly suitable for dense small-cells. The AC

Weiwei Zhou and Su Pan are with Nanjing University of Posts and Telecommunications (NJUPT), China. Gordon J. Sutton, J. Andrew Zhang and Ren Ping Liu are with University of Technology Sydney, Global Big Data Technologies Centre (GBDTC). Weiwei Zhou is also affiliated with UTS. Emails: Weiwei.zhou-1@ student.uts.edu.au; gordon.sutton@gmail.com; \{Andrew.Zhang; Renping.Liu\}@uts.edu.au; supan@njupt.edu.cn. policy is developed based on quantitative analysis for the distribution of the MAC delay and can guarantee the desired delay statistically. Our contributions of this paper are twofold. Firstly, we analyze the delay of tagged LAA eNBs using Gaussian approximation, based on the contention window, backoff stage and number of users. We adopt the exponential window size LBT scheme [1] where the backoff counter doubles at each collision, and also take into consideration the freezing time during the backoff process, distinguishing idle slots from busy slots. We analyze the backoff process on a multi-stage scheme, which is more fair for WiFi stations. Secondly, we derive the outage probability of the MAC delay, to indicate the QoS of the tagged station. Using a preset delay threshold, the system decides whether to admit a new incoming LAA eNB or to block it.

\section{SYSTEM MODEL}

We consider the LAA and WiFi coexisting systems in this paper where $n_{w} \geqslant 1 \mathrm{WiFi}$ stations share the same frequency channel with $n_{l} \geqslant 2$ LAA eNBs in the unlicensed band. We consider saturated traffic which is the worst case in communication. The corresponding MAC delay serves as an upper bound for other traffic models. We also assume that energy detection (ED) is used for both WiFi and LAA.

WiFi systems use the distributed coordination function (DCF) access mechanism based on Carrier Sensing Multiple Access (CSMA). If the channel is sensed idle for a time period of distributed interframe space (DIFS), the station will start a backoff time counter randomly chosen from $\left[0, W_{w}-1\right]$, where $W_{w}$ is the initial contention window size. The counter decreases by one for each time slot. If the channel is sensed busy during the backoff process, the decrement of the counter will freeze and restart after the channel becomes idle for another DIFS. When the counter reaches zero, the station will transmit data immediately. If the channel is idle, the transmission is successful and the process restarts. If the channel is busy, due to another station transmitting simultaneously, a collision occurs and the contention window size is doubled. The maximum backoff stage is $m_{w}$, the window size for stage $i$ is $W_{w, i}=2^{\min \left(i, m_{w}\right)} W_{w}$, and the maximum number of retransmission is $s_{w}$. The station should discard the packet or the frame after $s_{w}+1$ collisions.

The transmission attempt probability, $\tau_{w}$, that one WiFi station has data to transmit in a randomly chosen slot is [2]

$$
\tau_{w}=\frac{2\left(1-p_{w}^{s_{w}+1}\right)}{\left(1-p_{w}\right) \sum_{i=0}^{s_{w}}\left(W_{w, i}+1\right) p_{w}^{i}},
$$


where $p_{w}$ is the collision probability and can be obtained as

$$
p_{w}=1-\left(1-\tau_{l}\right)^{n_{l}}\left(1-\tau_{w}\right)^{n_{w}-1}
$$

For LBT, the LAA eNB competes for transmission opportunities through LBT. Once successful, the eNB will allocate the resource within this frame to multiple LTE users where both uplink (UL) and downlink (DL) slots can be included. UL access can be initiated by the UE, or it can be controlled by the eNB [1]. We consider the latter case, where the sensing period is typically very small and can be ignored when compared to the overall delay. These eNBs could be small-cell base stations of high density, therefore, there could be more than one eNBs competing for the time slots in the same frequency channel. In this paper, the LBT scheme is considered to have exponential backoff with $W_{l}$ initial contention window size, $m_{l}$ maximum backoff stages, and $s_{l}$ retransmission limits. An eNB transmits the whole frame in one transmission and needs to sense the channel again before the next frame transmission.

Similarly to $\mathrm{WiFi}$, the transmission probability for LAA eNBs $\tau_{l}$ and the collision probability $p_{l}$ can be expressed by

$$
\begin{gathered}
\tau_{l}=\frac{2\left(1-p_{l}^{s_{l}+1}\right)}{\left(1-p_{l}\right) \sum_{i=0}^{s_{l}}\left(W_{l, i}+1\right) p_{l}^{i}}, \\
p_{l}=1-\left(1-\tau_{w}\right)^{n_{w}}\left(1-\tau_{l}\right)^{n_{l}-1},
\end{gathered}
$$

where $W_{l, i}=2^{\min \left(i, m_{l}\right)} W_{l}$ is the window size of LAA eNB at stage $i$.

We now get a system model with key equations (1)-(4). In order to obtain $\tau_{w}, \tau_{l}, p_{w}$ and $p_{l}$ for the coexisting system, we need to jointly solve (1)-(4) which are dependent on each other and form a series of equations. Based on the Markov Chain method, this model can be applied to both uplink and downlink transmissions that share the same frequency channels.

\section{Delay-Guaranteed Admission Control}

In this section, we firstly analyze the probability distribution for MAC delay of the tagged stations, which are referred to as the new incoming LAA eNBs that wait to be admitted to the coexisting system. We then propose our admission control scheme based on the MAC delay analysis.

\section{A. Probability Distribution of MAC Delay}

MAC delay here refers to the time duration starting from the moment when a station generates a frame and triggers its initial backoff counter to that when the frame is successfully transmitted. The probability of the MAC delay for a tagged LAA eNB not exceeding $D$, when the successful transmission is made in stage $i$ after a total of $k$ backoff slots, can be computed as

$$
P(D ; i, k)=Q_{i}[k] \cdot \Phi(D \mid(i, k)),
$$

where $Q_{i}[k]$ is the probability that the successful transmission happens after backoff slot $k$ in stage $i$, and $\Phi(D \mid(i, k))$ is the conditional probability that MAC delays are no longer than $D$ given $i$ and $k$. Next, we show how to compute $Q_{i}[k]$ and $\Phi(D \mid(i, k))$.
1) Slots Analysis for Computing $Q_{i}[k]$ : Let $h_{i}[j]$ be the probability that a tagged LAA station selects $j$ as the initial backoff counter at backoff stage $i$,

$$
h_{i}[j]=\frac{1}{W_{l, i}}, 0 \leq i \leq s_{l}, 0 \leq j \leq W_{l, i}-1 .
$$

Consider the probability that a packet is successfully transmitted after $k$ backoff slots at stage $i$. It can be obtained by convolving all initial backoff counters before and at stage $i$, i.e., $\left(h_{0} \otimes h_{1} \otimes \ldots h_{i}\right)[k]$. The probability that the successful transmission of the tagged eNB occurs in stage $i$ is given by $w_{i}=\left(1-p_{l}\right) p_{l}^{i} /\left(1-p_{l}^{s_{l}+1}\right)$.

The probability that the tagged LAA eNB transmits a frame successfully after $k$ backoff slots in stage $i$ is obtained as

$$
Q_{i}[k]=w_{i} \cdot\left(h_{0} \otimes h_{1} \otimes \ldots h_{i}\right)[k] .
$$

2) Gaussian Approximation for Computing $\Phi(D \mid(i, k))$ : Let $d_{i, k}$ be the MAC delay when the tagged LAA eNB transmits a frame successfully after $k$ backoff slots and $i$ collisions, so that $d_{i, k} \sim N\left(\mu_{i, k}, \Sigma_{i, k}\right)$ according to the central limit theorem (CLT), when the number of time slots is large enough. The cumulative density function $\Phi(D \mid(i, k))=$ $P(d \leqslant D \mid i, k)$, which represents the probability of the delay being no longer than $D$ for successful transmission occurring after $k$ backoff slots in stage $i$, is given by

$$
\Phi(D \mid(i, k))=\frac{1}{2}\left(1+\operatorname{erf}\left(\frac{d_{i, k}-\mu_{i, k}}{\sqrt{2} \Sigma_{i, k}}\right)\right) .
$$

The mean $\mu_{i, k}$ and variance $\Sigma_{i, k}$ are computed by

$$
\mu_{i, k}=k \mu_{l}+i T_{c}+T_{s l}, \quad \Sigma_{i, k}=k \Sigma_{l},
$$

where $\mu_{l}$ and $\Sigma_{l}$ are respectively the mean and variance of the slot time when the tagged station is not transmitting, $T_{c}$ is the average slot duration for collisions involving the tagged LAA eNB, and $T_{s l}$ is the frame length for successful transmissions by the tagged LAA eNB. Use the superscript ' $b o$ ' to denote probabilities conditioned on the tagged eNB being in its backoff process and thus not transmitting. Next, we show how to compute $\mu_{i, k}$ and $\Sigma_{i, k}$.

The mean and variance of the slot period are obtained as

$$
\begin{aligned}
\mu_{l} & =P_{I d l e}^{b o} \delta+P_{s w}^{b o} T_{s w}+P_{s l}^{b o} T_{s l}+P_{c}^{b o} T_{c}^{b o}, \\
\Sigma_{l} & =P_{I d l e}^{b o} \delta^{2}+P_{s w}^{b o} T_{s w}^{2}+P_{s l}^{b o} T_{s l}^{2}+P_{c}^{b o}\left(T_{c}^{b o}\right)^{2}-\mu_{l}^{2},
\end{aligned}
$$

respectively, where $\delta$ is the idle slot duration; $T_{s w}$ is the frame length for WiFi stations; $T_{c}^{b o}$ is the average slot duration for collisions when the tagged LAA eNB is in backoff; and $P_{I d l e}^{b o}$, $P_{s w}^{b o}, P_{s l}^{b o}$ and $P_{c}^{b o}$ are respectively the probabilities that the channel is idle, a WiFi station transmits successfully, another LAA eNB transmits successfully, and that a collision occurs amongst the other nodes, all conditioned on the tagged LAA eNB not transmitting. These probabilities depend on $\tau_{w}$ and $\tau_{l}$, and can be computed as

$$
\begin{aligned}
P_{\text {Idle }}^{b o} & =\left(1-\tau_{w}\right)^{n_{w}}\left(1-\tau_{l}\right)^{n_{l}-1}, \\
P_{s w}^{b o} & =n_{w} \tau_{w}\left(1-\tau_{w}\right)^{n_{w}-1}\left(1-\tau_{l}\right)^{n_{l}-1}, \\
P_{s l}^{b o} & =\left(1-\tau_{w}\right)^{n_{w}}\left(n_{l}-1\right) \tau_{l}\left(1-\tau_{l}\right)^{n_{l}-2}, \\
P_{c}^{b o} & =1-P_{s w}^{b o}-P_{s l}^{b o}-P_{\text {Idle }}^{b o} .
\end{aligned}
$$


The parameter $T_{c}^{b o}$ can be calculated as

$$
\begin{aligned}
P_{2 W}= & \left(1-\left(1-\tau_{w}\right)^{n_{w}}-n_{w} \tau_{w}\left(1-\tau_{w}\right)^{n_{w}-1}\right)\left(1-\tau_{l}\right)^{n_{l}-1}, \\
P_{W L}= & \left(1-\left(1-\tau_{w}\right)^{n_{w}}\right)\left(1-\left(1-\tau_{l}\right)^{n_{l}-1}\right), \\
P_{2 L}= & \left(1-\tau_{w}\right)^{n_{w}}\left(1-\left(1-\tau_{l}\right)^{n_{l}-1}-\right. \\
& \left.\left(n_{l}-1\right) \tau_{l}\left(1-\left(1-\tau_{l}\right)^{n_{l}-2}\right)\right),
\end{aligned}
$$

and the corresponding durations due to collisions are $T_{s w}$, $\max \left\{T_{s w}, T_{s l}\right\}$ and $T_{s l}$, respectively. Combining these cases, $T_{c}^{b o}$ is given by

$$
T_{c}^{b o}=\frac{P_{2 W}}{P_{c}^{b o}} T_{s w}+\frac{P_{W L}}{P_{c}^{b o}} \max \left\{T_{s w}, T_{s l}\right\}+\frac{P_{2 L}}{P_{c}^{b o}} T_{s l} .
$$

$T_{c}$ is similarly obtained as

$$
T_{c}=T_{s l}+\left(\max \left\{T_{s w}, T_{s l}\right\}-T_{s l}\right)\left[1-\left(1-\tau_{w}\right)^{n_{w}}\right] / p_{l} .
$$

Substituting (12)-(16) into (10) and (11) generates $\mu_{l}$ and $\Sigma_{l}$. We can then obtain $\mu_{i, k}$ and $\Sigma_{i, k}$ by substituting (17) into (9), and finally get $\Phi(D \mid(i, k))$ through (8).

\section{B. Admission Control Policy}

We define the Outage Probability of delay, $P_{\text {out }}$, as the probability that the delay of the tagged station exceeds a desired threshold, which is given by

$$
P_{\text {out }} \triangleq P\left\{d>D_{t h}\right\}=1-\sum_{i=0}^{s} \sum_{k=0}^{L_{i}-1} P(D ; i, k),
$$

where $d$ is the MAC delay of the tagged LAA eNB; $L_{i}$ is the length of $Q_{i}$; and $D=D_{t h}$ is the preset delay threshold representing the maximum MAC delay that the tagged eNB could tolerate.

If the MAC delay of the new incoming LAA eNB exceeds this threshold, it should not be admitted into the system. Otherwise, QoS may not be guaranteed and the performance of other stations will also degrade if the tagged incoming station is admitted. Generally, the outage probability shall be no more than $5 \%$, which means $P_{\text {out }} \leqslant 0.05$.

We show the proposed Admission Control policy together with the detailed process for computing the Outage Probability of delay in Algorithm 1. We also summarize the general idea as follows:

1) For the given $n_{w}, n_{l}$, where $n_{l}$ should include the tagged new incoming LAA eNB requesting for admission, compute $\tau_{w}, p_{w}, \tau_{l}$ and $p_{l}$;

2) Calculate the outage probability $P_{\text {out }}$, with the preset delay threshold $D_{t h}$;

3 ) If $P_{\text {out }}$ is not greater than 0.05 , the system should admit the tagged LAA eNB; otherwise, deny its request, so as to guarantee the QoS of existing users.

\section{Simulation Results}

We set the frame length for LAA eNBs as $T_{s l}=8 \mathrm{~ms}$ when there is WiFi traffic [5], which conforms to the access priority class 3 in the downlink of 3GPP's LAA. Users within one service class can be assumed to have the same delay requirement. For a different type of user, the parameters, $W_{0}$,

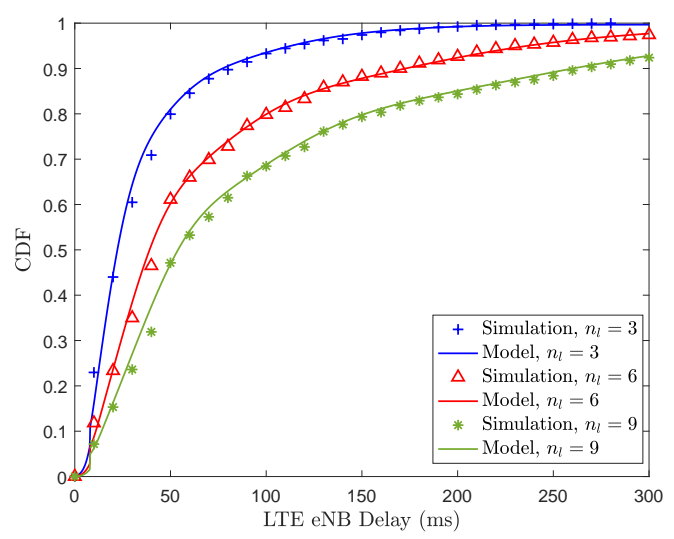

Fig. 1. Cumulative density function of the MAC delay of the tagged LAA eNB when $n_{w}=6$ and $T_{s w}=271 \mu s$.

$m^{\prime}, T_{s l}$, etc., should conform to a certain access priority class according to [1]. Class-based services are adopted in both $\mathrm{WiFi}$ (in IEEE 802.11e) and LTE (defined in QCI). To simplify the analysis, we assume that all the users served by LAA eNBs are in the same priority class.

We simulate the coexisting system with $6 \mathrm{WiFi}$ stations and 3,6 or 9 LAA eNBs, including the tagged LAA eNB requesting admission, i.e. $n_{w}=6, n_{l}=3,6$, or 9 . The number of time slots is $10^{6}$ in our simulation. The payload size of $\mathrm{WiFi}$ is usually 1500 bytes so that $T_{s w}=271 \mu s$ when data rate is $72.2 \mathrm{Mbps}$ [2]. To assess the fairness between WiFi and LAA systems, we consider WiFi also operating with access priority class 3, noting that $271 \mu \mathrm{s}$ is within the MCOT, and consider $T_{s w}$ of $271 \mu \mathrm{s}, 1 \mathrm{~ms}, 2 \mathrm{~ms}$ and $8 \mathrm{~ms}$. We set $D_{t h}=0.3 \mathrm{~s}$, and for any other type of services, $D_{t h}$ can be adjusted according to the delay requirement of the services. Other parameters are $W_{w}=16, m_{w}=5, s_{w}=7$ for WiFi stations; $W_{l}=16$, $m_{l}=2, s_{l}=4$ for LAA eNBs; and $\delta=9 \mu$ s for the coexisting systems.

Fig. 1 shows the cumulative density functions of the MAC delay of the tagged LAA eNB for the simulation and model

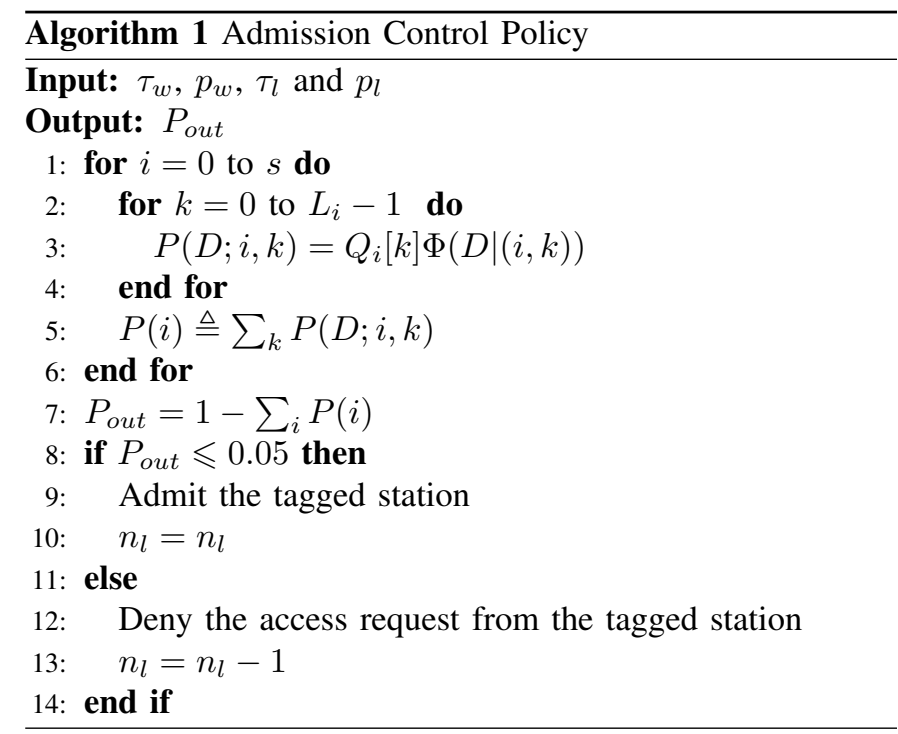




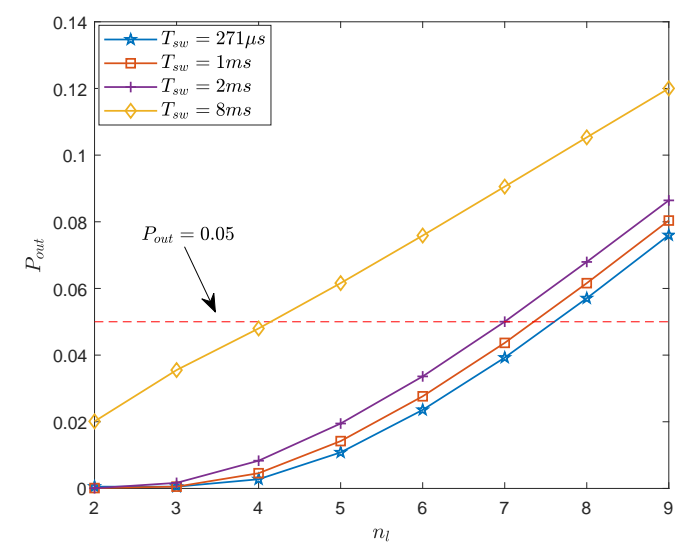

Fig. 2. Outage probability for tagged LAA stations with different WiFi frame length when $n_{w}=6 \mathrm{WiFi}$ stations are already present.

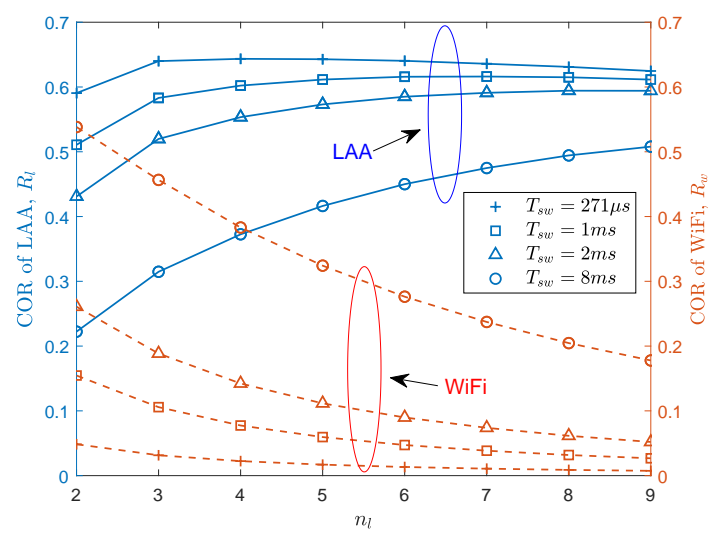

Fig. 3. CORs of the LAA and WiFi systems with different WiFi frame lengths when $n_{w}=6$ and $D_{t h}=0.3 \mathrm{~ms}$.

results. The functions from the analytical Gaussian model match those from the simulation very well, especially for larger delay values. In Fig. 2, we demonstrate how the number of admitted LAA eNBs impacts the outage probability. From the figure, we can observe that the outage probability increases rapidly when the number of LAA eNB grows. The system can admit 7 LAA eNBs at most when WiFi frame length is $271 \mu \mathrm{s}$ and $1 \mathrm{~ms}$ while admitting $6 \mathrm{LAA}$ eNBs when $T_{s w}$ increases to $2 \mathrm{~ms}$, and $4 \mathrm{LAA}$ eNBs when $T_{s w}$ is $8 \mathrm{~ms}$.

We define the channel occupation ratio $(C O R)$ as the proportion of successful transmission time occupied by either LAA or WiFi. Let $R_{w}$ and $R_{l}$ be the CORs for WiFi stations and LAA eNBs respectively. Fig. 3 shows the CORs when $n_{w}=6$. The figure indicates that the LAA system occupies most of the wireless resource due to the longer frame length than WiFi. We notice that the COR for LAA does not always increase with $n_{l}$, as more LAA eNBs cause more collisions. Moreover, the channel utility $R_{w}+R_{l}$ does not always increase when the number of the admitted LAA increases.

We also investigate the fairness of channel occupation between WiFi and LAA systems by defining the fairness index of CORs as $\eta=\left(\left(R_{w}+R_{l}\right)^{2}\right) / 2\left(\left(R_{w}^{2}+R_{l}^{2}\right)\right)$ [6]. The relationship between the fairness index and the number of admitted LAA

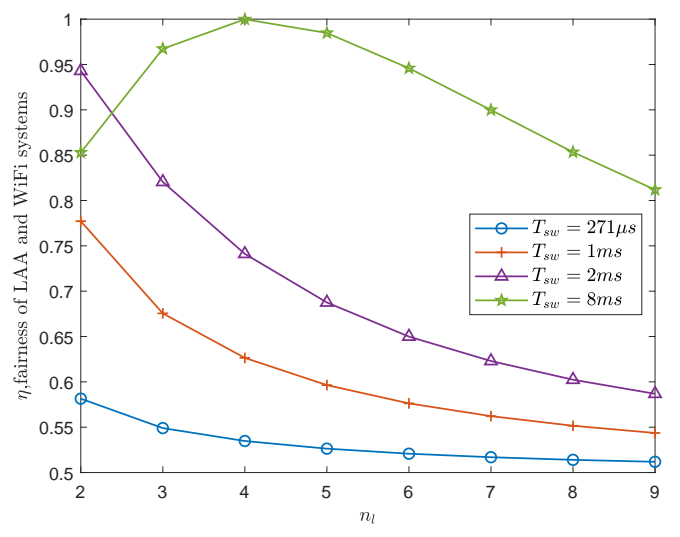

Fig. 4. Fairness of LAA and WiFi systems when $n_{w}=6$ and $D_{t h}=0.3 \mathrm{~ms}$.

eNBs with different WiFi frame lengths is demonstrated in Fig. 4. The fairness index improves significantly when the WiFi frame length increases from $271 \mu \mathrm{s}$ to $8 \mathrm{~ms}$, which indicates that the frame length has a considerable impact on the fairness for the coexisting system. The CORs and fairness can be adjusted by controlling the number of admitted LAA eNBs.

\section{CONClusion}

In this paper, we proposed an admission control scheme for LAA eNBs based on quantitative delay analysis for LAA and WiFi coexisting systems in the unlicensed band. Our scheme can guarantee the desired MAC delay for the LAA eNBs via controlling the number of admitted LAA eNBs, based on the number of existing WiFi stations. Our algorithm also works for other systems, such as MulteFire, that operate in the unlicensed band and supporte the LBT mechanism. Our investigation on channel occupation ratio (COR) discloses that LAA eNBs benefit from designated longer frame and that admitting more eNBs can degrade the overall system performance. Our study on the fairness index of COR further highlights the importance of the frame length and the number of admitted stations on the overall system fairness of access. Our work hence provides an accurate method for admission control, guaranteeing the MAC delay of the LAA eNBs.

\section{REFERENCES}

[1] ETSI, "LTE; Evolved Universal Terrestrial Radio Access (E-UTRA); physical layer procedures (3GPP TS 36.213 version 14.5.0 Release 14)," Tech. Rep. 417-427, 2018.

[2] G. J. Sutton, R. P. Liu, and Y. J. Guo, "Delay and reliability of load-based listen-before-talk in LAA," IEEE Access, vol. 6, pp. 6171-6182, 2018.

[3] R. Yin, G. Yu, A. Maaref, and G. Y. Li, "LBT-based adaptive channel access for LTE-U systems," IEEE Transactions on Wireless Communications, vol. 15, no. 10, pp. 6585-6597, Oct 2016.

[4] Q. Zhang, Q. Wang, Z. Feng, and T. Yang, "Design and performance analysis of a fairness-based license-assisted access and resource scheduling scheme," IEEE Journal on Selected Areas in Communications, vol. 34, no. 11, pp. 2968-2980, 2016.

[5] M. Mehrnoush, S. Roy, V. Sathya, and M. Ghosh, "On the Fairness of Wi-Fi and LTE-LAA Coexistence," IEEE Transactions on Cognitive Communications and Networking, pp. 1-1, 2018.

[6] R. Jain, D.-M. Chiu, and W. R. Hawe, A quantitative measure of fairness and discrimination for resource allocation in shared computer system. Eastern Research Laboratory, Digital Equipment Corporation Hudson, MA, 1984, vol. 38 . 